\title{
APPLICATION OF THE COPPER-ZINC COUPLE TO THE ESTIMATION OF NITRATES IN WATER.
}

By Robert Brewer Lee, B.Sc., F.S.C.,

Of Birkbeck Laboratory, Universal College, London.

Some time ago $I$ had occasion to consider the most readily available methods of estimating nitrates in the process of water analysis.

Crum's method by reduction to nitric oxide was found most satisfactory for regular use in the laboratory; but we were also in need of a handy method applicable in circumstances where few of the appliances of an analytical laboratory were accessible.

In the Journal of the Chemical Society, vol. xxxix, page 100, Mr. Whiteley Williams describes a process of reducing the nitric acid to ammonia by a copper-zinc conple, and nesslerising a few cubic centimetres of the water so treated.

On endeavouring to repeat Mr. Williams' experiment, only inaccurate results were obtained. After trying various modifications of the method, I came to the conclusion that the following are the conditions of greatest accuracy.

1. The nitric acid should only be present in small quantity-best not more than $\mathbf{1 0}$ or 12 grains per gallon. Waters containing more than this should be proportionately diluted with distilled water.

2. The couple is most active in slightly acid solutions. I find it best to acidify with oxalic acid, which has the advantages both of precipitating the lime, and of forming an insoluble compound with the zinc.

The method of procedure is as follows:-The couple is made by immersion of clean zinc foil in a 3 per cent. solution of copper sulphate for 10-15 minutes. It is then gently washed, and about 1 square decimetre placed in a wide-mouthed stoppered bottle of $300-$ 400 c.c. capacity. About 0.5 gramme of oxalic acid is added, and the bottle filled with the water to be analysed. The reduction may then safely be assumed to take place ii the cold in 24 hours. But if the bottle be heated in a water-brth to $55^{\circ}-60^{\circ} \mathrm{C}$. the reduction will be found to be completed in $1 \frac{1}{2}$ to 2 hours.

From 2 to 10 c.c. of the water are now carefuily wilhdıawn in a graduated pipette, made up to 50 c.c. in the Nessler glass with ammonia-free water, and nesslerised in the usual way.

The use of oxalic acid enables the temperature to be raised to $60^{\circ} \mathrm{C}$. without loss of ammonia, and the reduction is then completed rapidly. The oxalic acid used must of course be free from ammonia and nitric acid.

Attempts were made to use granulated zine instead of zinc foil for making the couple; but the couple so obtained was woaker and more uncertain in its action.

The following are the results of the experiments made. When not otherwise stated, I worked upon dilute solutions of potassium nitrate of known strength; but in the case of natural waters the figures obtained are compared with determinations by Crum's method.

As the work was with a view to water analysis, the results are stated in grains per gallon of nitric acid $\left(\mathrm{N}_{2} \mathrm{O}_{5}\right)$. 
In the first seven experiments, granulated zine was employed for making the couple, and the quantity of oxalic acid varied from 1 to 2 grams.
$\mathrm{N}_{2} \mathrm{O}_{s}$ present.
$\mathrm{N}_{2} \mathrm{O}_{5}$ found.
Remarks.

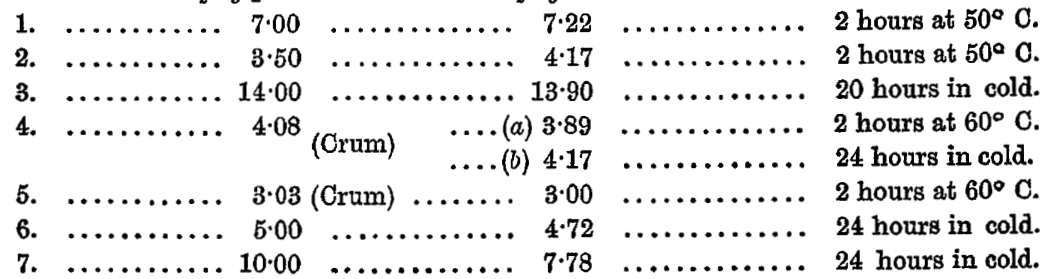

After this, zine foil was employed.
8. ........... 7.00
6.95

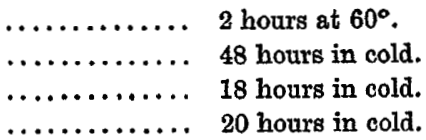

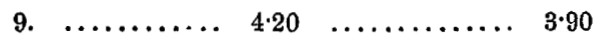

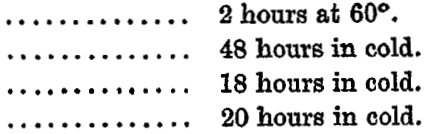
10. $\ldots \ldots \ldots \ldots \ldots, 5 \cdot 00 \quad \ldots \ldots \ldots \ldots \ldots, \quad 5.28$
11. $\ldots \ldots \ldots \ldots, 1.00 \ldots \ldots \ldots \ldots ., 95$
20 hours in cold.
12. ..........6.62.26 (Crum) $\ldots \ldots \ldots .443 .36$
The water was diluted
to 10 times its original
volume, then stood on
couple 20 hours in cold.
14. $\ldots \ldots \ldots \ldots \ldots$ 17.90 (Crum) $\ldots \ldots \ldots . .15 .56$
40 hours in cold.

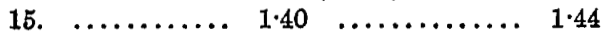
................. 2 hours at $55^{\circ}-60^{\circ} \mathrm{C}$.
16. $\ldots \ldots \ldots \ldots . .4 .20 \quad \ldots \ldots \ldots \ldots . .4 .17$
$\ldots \ldots \ldots \ldots \ldots . .2$ hours at $55^{\circ}-60^{\circ} \mathrm{C}$.
17. $\ldots \ldots \ldots \ldots \ldots$ 5 18 (Crum) $\ldots \ldots \ldots$ 5.45

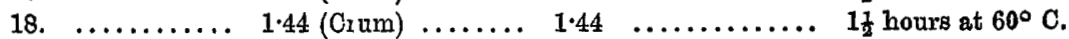

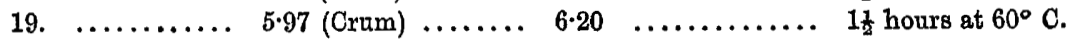

In experiments 8 to 14 the quantity of oxalic acid varied from 0.5 to $1.0 \mathrm{gram}$; and in the last 5 experiments it was 0.5 gram.

In conclusion, I wish to record my obligations to Dr. Graham, in whose laboratory these experiments were made.

\section{MILK ANALYSIS IN BOSTON, U.S.A.}

Is connection with extracts from Dr. Bell's new book on Milk Analysis, \&c., printed on another page, the following analyses made during one year, by the Analyst of Boston, will, no doubt, be of interest to our readers, as showing the standard adopted in that city.

Milix Analyses made During the Year.

\begin{tabular}{|c|c|c|c|c|c|c|c|}
\hline No & Gravity. & $\begin{array}{l}\text { Oream } \\
\text { per cent. }\end{array}$ & $\begin{array}{l}\text { Total } \\
\text { solids. }\end{array}$ & $\underset{\text { matter }}{\text { Fatty }}$ & $\begin{array}{l}\text { Solida } \\
\text { not fat. }\end{array}$ & Water. & $\begin{array}{l}\text { Per cent. of } \\
\text { water added. }\end{array}$ \\
\hline 1 & $1 \cdot 028$ & $1 \frac{1}{2}$ & $9 \cdot 20$ & 0.42 & $8 \cdot 78$ & $90 \cdot 80$ & 15 \\
\hline 2 & " & $3 \frac{1}{2}$ & $7 \cdot 85$ & 098 & $6 \cdot 87$ & $92 \cdot 15$ & 35 \\
\hline 3 & ", & $5^{2}$ & $10 \cdot 50$ & 1.45 & $9 \cdot 05$ & $89 \cdot 50$ & 20 \\
\hline 4 & " & 8 & $12 \cdot 80$ & $2 \cdot 15$ & $10 \cdot 65$ & $87 \cdot 20$ & \\
\hline 5 & ," & 2 & $7 \cdot 42$ & 0.58 & $6 \cdot 84$ & $92 \cdot 58$ & 40 \\
\hline 6 & ", & $4 \cdot 5$ & $11 \cdot 15$ & $1 \cdot 46$ & 969 & $88 \cdot 85$ & 15 \\
\hline 7 & $"$ & 4 & $9 \cdot 50$ & $1 \cdot 32$ & $8 \cdot 18$ & $90 \cdot 50$ & 25 \\
\hline 8 & ", & $4 \cdot 5$ & $11 \cdot 20$ & $1 \cdot 68$ & $9 \cdot 52$ & $88 \cdot 80$ & 15 \\
\hline 9 & $"$ & 7 & $10 \cdot 98$ & $2 \cdot 15$ & $8 \cdot 83$ & $89 \cdot 02$ & 15 \\
\hline 10 & $"$ & 5 & $10 \cdot 25$ & $1 \cdot 65$ & $8 \cdot 60$ & $89 \cdot 75$ & 20 \\
\hline 11 & $"$ & 7 & $11 \cdot 15$ & $2 \cdot 15$ & $9 \cdot 00$ & $88 \cdot 85$ & 15 \\
\hline 12 & ", & $7 \cdot 5$ & 13.70 & $2 \cdot 18$ & $11 \cdot 52$ & 86.30 & pure. \\
\hline 13 & ", & 6 & 1025 & 1.92 & 883 & $89 \cdot 75$ & 20 \\
\hline 14 & $"$ & 4 & 1087 & $1 \cdot 80$ & $9 \cdot 07$ & $89 \cdot 13$ & 16 \\
\hline 15 & $"$ & 5 & $10 \cdot 40$ & 1.58 & $8 \cdot 82$ & 8960 & 20 \\
\hline 16 & $"$ & 7 & $10 \cdot 60$ & $1 \cdot 95$ & $8 \cdot 65$ & $89 \cdot 40$ & 20 \\
\hline 17 & $"$ & 9 & 10.45 & $2 \cdot 65$ & 780 & 89.55 & 20 \\
\hline 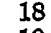 & " & 6.5 & $10 \cdot 40$ & $1 \cdot 82$ & $8 \cdot 58$ & $89 \cdot 60$ & 20 \\
\hline 19 & " & 7 & 11.05 & $2 \cdot 19$ & $8 \cdot 86$ & $98 \cdot 95$ & 16 \\
\hline
\end{tabular}




\section{FOOD ADULTERATION IN FRANCE.}

The following Analyses were made at the Paris Municipal Chemical Laboratory, during the month of June, 1883 :-

\begin{tabular}{|c|c|c|c|c|c|c|c|c|c|}
\hline 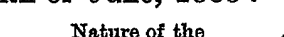 & & & & & & a.. & & & \\
\hline Samples Analysed. & Good. & & Passable. & & Injur & & Injurio & & Totals. \\
\hline Wines ........... & 71 & .. & 57 & .. & 412 & .. & $1 \dot{3}$ & .. & 553 \\
\hline Vinegars...$\ldots \ldots \ldots$ & 1 & .. & 3 & .. & 1 & .. & - & .. & $\mathbf{5}$ \\
\hline Beers $\ldots \ldots \ldots \ldots \ldots$ & 16 & .. & - & .. & 2 & .. & 3 & .. & 21 \\
\hline Ciders...$\ldots \ldots \ldots \ldots$. & - & .. & 1 & .. & $\mathbf{5}$ & .. & - & .. & 6 \\
\hline Alcohols and Liqueurs & - & .. & 2 & .. & - & .. & 12 & .. & 14 \\
\hline Syrups.............. & 1 & .. & - & .. & - & .. & - & .. & 1 \\
\hline Waters $\ldots . . . . . . . .$. & 15 & .. & $\mathbf{3}$ & .. & 2 & .. & 21 & .. & 41 \\
\hline Milks.$\ldots \ldots \ldots \ldots \ldots$ & 44 & .. & 173 & .. & 197 & .. & - & .. & 414 \\
\hline Malt................ & - & .. & - & .. & - & .. & - & .. & - \\
\hline Buttere $\ldots \ldots \ldots \ldots$ & 15 & .. & - & .. & 2 & .. & - & .. & 17 \\
\hline Oils $\ldots \ldots \ldots \ldots \ldots$ & 3 & .. & 1 & .. & 4 & .* & - & .. & 8 \\
\hline Flotars ............... & 5 & .. & - & .. & 8 & .. & - & .. & 13 \\
\hline Dough, Bread......... & 1 & .. & 1 & .. & - & .. & 1 & .. & $\mathbf{B}$ \\
\hline Sweetmeats........... & - & .. & 一 & .. & - & .. & 1 & .. & 1 \\
\hline Meats .............. & - & .. & - & .. & 1 & .. & - & .. & 1 \\
\hline Preserves.............. & $\mathbf{3}$ & .. & - & .. & - & .. & 2 & .. & 5 \\
\hline Salt, Pepper ......... & 5 & .. & - & .. & 13 & .. & - & .. & 18 \\
\hline Chicory, Coffee, Tea .. & - & .. & 2 & .. & - & .. & 一 & .. & $\mathbf{z}$ \\
\hline Chocolates .......... & 1 & .. & - & .. & 7 & .• & - & .. & 8 \\
\hline Honeys.......... & - & . & 一 & .. & - & .. & 一 & -・ & - \\
\hline Confitures...$\ldots \ldots$. & - & .. & - & .. & - & .. & - & •. & - \\
\hline Colouring Materials .. & 2 & .. & 2 & .. & - & .. & $\mathbf{5}$ & .. & 9 \\
\hline Toys $\quad \ldots \ldots . . . . . . .$. & - & .. & - & .. & - & .. & 7 & .. & 7 \\
\hline Coloured Papers .... & - & .. & - & .. & - & .. & - & .. & - \\
\hline Tins................ & 2 & .. & - & .. & 1 & .. & - & .. & 3 \\
\hline Spices...$\ldots \ldots \ldots \ldots$ & 8 & .. & - & .. & - & . & - & .. & 8 \\
\hline 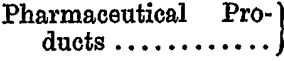 & \}- & • & - & $\cdot \cdot$ & - & $\cdots$ & - & $\cdots$ & 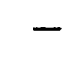 \\
\hline Perfumery .......... & 5 & .. & 一 & .. & - & .. & 一 & $\cdots$ & 5 \\
\hline Various ............. & 22 & *. & 2 & .. & 8 & ・・ & 11 & $\cdots$ & 43 \\
\hline Total.... & & & 247 & & 663 & & 76 & & 206 \\
\hline
\end{tabular}

